\title{
Modelos de atenção à saúde e sua relação com a gestão de enfermagem hospitalar
}

\author{
Health care models and their relationship with hospital nursing management
}

Modelos de atención de la salud y su relación con la gestión de enfermería hospitalaria

\author{
Laura Andrian Leal'; Silvia Helena Henriques"; Lana Jocasta de Souza Brito ${ }^{\prime \prime \prime}$; \\ Lázaro Clarindo Celestino ${ }^{\prime v}$; Daniela Sarreta Ignáciov; Aline Teixeira Silva ${ }^{v I}$
}

\begin{abstract}
RESUMO
Objetivo: refletir sobre os modelos de atenção e suas relações com o trabalho em enfermagem hospitalar. Método: estudo reflexivo realizado em junho de 2019, a partir do aprofundamento das leituras, discussão dos dados e experiência das autoras, conduzido por duas correntes: Considerações históricas sobre o hospital e Modelo de atenção e enfermagem hospitalar. Resultados: verificou-se que o modelo tradicional de gestão ainda está arraigado à vida institucional, contudo, considerar suas limitações é essencial, fazendo-se necessária a transposição para um modelo que agregue o coletivo de trabalhadores, de modo que se sintam engajados e motivados por se considerarem corresponsáveis e fundamentais no processo de trabalho. Conclusão: é necessário a incorporação de um modelo de gestão mais participativo, com o envolvimento da equipe multiprofissional na tomada de decisão e resolução dos problemas, bem como na elaboração de propostas de melhorias.

Descritores: Enfermagem; hospitais; administração hospitalar; recursos humanos.
\end{abstract}

\section{ABSTRACT}

Objective: to think about care models and their relationship to working in hospital nursing. Method: this reflective study was conducted in June 2019 on the basis of the authors' in-depth reading, discussions of data and experience, guided by two approaches: historical considerations about the hospital, and the model of hospital care and nursing. Results: the traditional management model was found to be still deeply rooted in institutional life, although it is essential to consider its limitations, and necessary to move on to a model that aggregates the workers into a whole, so that they feel engaged and motivated by feeling co-responsible in, and fundamental to, the work process. Conclusion: it is necessary to introduce a more participatory management model, involving multi-professional teams in decision making and problem solving, as well as in formulating proposals for improvement.

Descriptors: Nursing; hospitals; hospital administration; human resources.

\section{RESUMEN}

Objetivo: reflexionar sobre los modelos de atención y su relación con el trabajo en enfermería hospitalaria. Método: estudio reflexivo realizado en junio de 2019, basado en lecturas, la discusión de datos y la experiencia de las autoras, realizado por dos corrientes: Consideraciones históricas sobre el hospital y Modelo de atención y enfermería hospitalaria. Resultados: se verificó que el modelo de gestión tradicional todavía está enraizado en la vida institucional, sin embargo, tener en cuenta sus limitaciones es esencial, y se hace necesario transponerlo a un modelo que agregue al colectivo de trabajadores, para que se sientan comprometidos y motivados por considerarse corresponsables y fundamentales en proceso de trabajo. Conclusión: es necesario incorporar un modelo de gestión más participativo, con la implicación del equipo multiprofesional en la toma de decisiones y la resolución de problemas, así como en la elaboración de propuestas de mejora.

Descriptores: Enfermería; hospitales; administración hospitalaria; recursos humanos.

\section{INTRODUÇÃO}

O trabalho, na contemporaneidade, vem sofrendo transformações decorrentes da globalização, que tem provocado inovações tecnológicas, e mudanças nos processos de trabalho. Assim, buscar um perfil independente, reativo e empreendedor gerou a necessidade de desenvolver competências relacionadas aos diversos campos profissionais, conforme exigências solicitadas para cada área, que devem impactar diretamente na sua saúde ${ }^{1}$.

Em particular, no cenário da América Latina, o trabalho sofre o impacto das políticas de recorte neoliberal, como uma forma capitalista avançada. Nessa realidade, estão presentes a redução da força de trabalho, o desemprego, a competitividade e a consequente busca por novas formas de organização dos processos de trabalho². Ainda há que se dizer que a fim de garantir a competitividade, melhores produtos e serviços, as organizações tendem a buscar novos modelos de atenção e tecnologia. 
Na área da saúde esta realidade não é diferente. Os avanços nos cenários das práticas de saúde, especialmente no âmbito hospitalar, têm repercutido na organização do trabalho. No hospital, organização de prestação de serviços de saúde onde congregam saberes, profissionais, tecnologia e infra-estrutura diversificados, o trabalhador é o sujeito da sua própria ação e da sua relação com o meio ${ }^{3}$. Nesse contexto, observa-se que o volume de atividades, diversas categorias, condições laborais, bem como as formas de organização e modelos de atenção à saúde podem interferir no processo de trabalho da enfermagem.

Modelos assistenciais, modelos de atenção ou modos de intervenção em saúde são entendidos como diferentes combinações tecnológicas com diferentes finalidades, como resolver problemas e atender necessidades de saúde, em determinada realidade e população adstrita (indivíduos, grupos, ou comunidades), organizar serviços de saúde ou intervir em situações, em função do perfil epidemiológico e da investigação dos danos e riscos à saúde ${ }^{4}$.

Em se tratando de modelos de atenção focados em instituições hospitalares, pesquisadores descrevem que a atenção hospitalar, vem sendo construída a partir de elementos normativos que confluem para a consolidação de uma rede articulada de serviços de saúde. No entanto, ao mesmo tempo, se alinha a uma concepção gerencialista ${ }^{5}$.

Os impactos que os modelos de atenção causam nos profissionais da saúde, especialmente na enfermagem podem, em algumas vezes, ser negativos, pois requerem um processo contínuo de adaptação dos modos de cuidar frente às complexidades cada vez maiores, identificadas. Observa-se também que diferentes modelos assistenciais implicam em dinâmicas organizacionais diversas, impactando nas cargas de trabalho, podendo desencadear desgaste no trabalhador ${ }^{6}$.

Destaca-se a natureza do espaço hospitalar, fortemente marcado por modelos verticalizados, focados na doença e no tratamento, permeado por pactuações para o desenvolvimento do trabalho que colocam os médicos em posição privilegiada, com baixa interlocução com outras áreas, e ainda, em relação aos profissionais de enfermagem nota-se a visão fragmentada dos usuários, além de questões como a falta de isonomia salarial ${ }^{7}$. Assim, modelos de atenção aos usuários dos serviços podem repercutir de forma positiva ou negativa na organização do trabalho dos profissionais de enfermagem.

Gestores têm o papel crucial na organização do trabalho, na implantação de modelos de atenção flexíveis e participativos, que criem oportunidades aos trabalhadores, dando-lhes maior responsabilidade, autonomia no processo decisório, nos resultados, no seu desenvolvimento pessoal e profissional e, consequentemente provocando um efeito positivo para o cuidado prestado ao usuário.

Frente ao exposto, este estudo vislumbra refletir sobre modelos de atenção e sua relação com o trabalho em enfermagem hospitalar. Para tal, iniciamos fazendo breve consideração histórica sobre o método de organização do trabalho no hospital para posteriormente refletir em como o modelo de atenção pode interferir na gestão dos serviços de enfermagem.

\section{CONSIDERAÇÕES HISTÓRICAS SOBRE O HOSPITAL}

Os hospitais são organizações cujo papel vem se modificando e expandindo, na perspectiva de responder às transformações sociais de cada época. Por muito tempo, foi um local filantrópico, caritativa e espiritual, destinado à exclusão social. Os agentes que atuavam nos hospitais eram religiosos e/ou leigos que prestavam assistência para garantir a sua salvação espiritual. A doença ocupava o espaço doméstico, a prática médica consistia na relação entre o doente e o médico, único agente desse trabalho ${ }^{8}$.

Em decorrência de necessidades que emergiram com a revolução industrial, as fábricas requeriam trabalhadores saudáveis para desenvolver o processo produtivo. Assim, a partir do final do século XVIII e começo do século XIX iniciou-se a reorganização e reforma dos hospitais, que se tornaram instrumentos terapêuticos, tendo como foco a força de trabalho incapacitada pela doença, com a finalidade de repô-la no processo produtivo. Em síntese, o modelo clínico de atendimento à saúde apoiava-se na análise do funcionamento do organismo, procurando as causas dos distúrbios para restabelecê-lo . $^{\circ}$

Com a reorganização, o hospital tornou-se espaço de ensino e de experimentação de saberes e práticas médicas, constituindo-se também em local para tratar o corpo doente em sua dimensão individual sendo o médico, agente basilar do trabalho neste modelo de atenção, do qual decorriam as ações de um conjunto de outros agentes, entre eles, de enfermagem.

Enquanto o modelo clínico de atenção foi se consolidando, a administração dos hospitais deixava de ser eclesiástica e a organização do trabalho em saúde também foi se conformando, a partir das teorias científica e clássica. Nesse sentido, a enfermagem, para organizar o trabalho e adotar estratégias racionalizadoras, ancorou-se também nos pressupostos de Taylor e Fayol, que conformam o método de organização do trabalho, denominado método funcional, com ênfase no domínio de tarefas, exprimindo somente um aspecto técnico da divisão do trabalho, bem como a divisão social existente na enfermagem ${ }^{9}$. 
Esta forma de organizar o trabalho, por meio de sua divisão, provocou também a divisão do homem, impossibilitando-o de gerir suas ações, uma vez que foi introduzido no processo de trabalho um novo elemento especializado, o gerente. A gerência é incorporada tendo como meta e preocupação fazer valer a autoridade, a disciplina, a hierarquia, a responsabilidade, o planejamento, o controle e a coordenação.

Todas as características da organização do trabalho industrial tanto do ponto de vista técnico (das tarefas na visão de Taylor) quanto de estrutura organizacional (Fayol) foram introduzidas e incorporadas na organização do trabalho hospitalar a partir da década de 20, consolidadas ao longo do século XX e, ainda se fazem presentes. Taylor contribuiu com uma série de princípios e características de administrar que possibilitaram aproximar a gestão da ciência. Por meio da administração científica, aumenta a produtividade, contribuindo para a eficiência e aumento dos salários dos trabalhadores ${ }^{10}$. Já Fayol enfatiza a unidade de direção e comando, os incentivos não financeiros, a descentralização e a coordenação, que têm grande relevância ainda hoje.

De qualquer modo, a Abordagem Clássica da Administração recebeu críticas por se caracterizar como um meio de exploração dos trabalhadores para se alcançar o máximo de produtividade para as empresas. Assim, na tentativa de corrigir essa desumanização surge na década de 20 a teoria das relações humanas na tentativa de valorizar o capital humano e suas inter-relações, valores e comportamento de grupo ${ }^{11}$. Entretanto, críticas a essa Teoria também foram apontadas, como a valorização das relações informais desconsiderando as relações formais.

Em função das críticas feitas tanto à abordagem clássica, pelo seu tecnicismo, como à abordagem humanística, pelo seu romantismo, desenvolveu-se, na década de 40, a teoria da burocracia, idealizada por Max Weber. Para este filósofo ${ }^{12}$, a burocracia é a organização eficiente e apresenta características como o caráter legal das normas e regulamentos, formalidade das comunicações, caráter racional e divisão do trabalho, hierarquia de autoridade, procedimentos padronizados, competência técnica e meritocracia, visando à máxima eficiência da organização.

Assim como as demais teorias principais que influenciaram a organização do trabalho nas instituições de saúde, a Teoria da Burocracia foi muito criticada por desconsiderar as relações informais, além do sistema rígido de controle e de autoridade.

Apesar das críticas, há que se ressaltar que a organização do trabalho e o gerenciamento no setor saúde, especialmente no âmbito hospitalar, constituíram-se, historicamente, sob influência do modelo Taylorista da administração clássica e do modelo burocrático, trazendo implicações para a organização do trabalho de enfermagem, principalmente sob o âmbito da fragmentação do trabalho, na execução e divisão das tarefas, na hierarquização, sistematização e departamentalização, ou seja, os trabalhadores passaram a ter que cumprir suas tarefas de forma individualizada e no menor tempo possível.

\section{MOdELO DE ATENÇÃO E ENFERMAGEM HOSPITALAR}

O hospital constitui-se em uma organização social complexa, e para desenvolver suas atividades utiliza sofisticadas tecnologias, extensa divisão de trabalho entre as diversas categorias profissionais, aliadas a um amplo sistema de coordenação de tarefas e funções ${ }^{13}$. Caracteriza-se pela forte concentração de poder decisório no topo da estrutura, grande número de níveis hierárquicos dificultando o fluxo da comunicação e informação. Estes são elementos ainda presentes nas estruturas organizacionais que tem viabilizado relações de trabalho formalizadas e conflituosas e, restringindo as possibilidades de participação e de articulação do conjunto de profissionais.

O processo de gestão nessas instituições encontra-se ainda fortemente alicerçado no modelo clínico de atenção à saúde, cujos saberes são aqueles oriundos da anatomia, fisiologia, biologia, patologia e farmacologia. Além disso, ainda hoje o hospital constitui-se uma organização subordinada a uma gestão voltada para o controle, padronização e rotinização do processo de trabalho.

A lógica do modelo de gestão tradicional, pautado nas teorias de Taylor e Fayol, bem como na teoria da burocracia de Weber, impõe que o processo de trabalho da equipe de enfermagem no ambiente hospitalar seja marcado pela fragmentação do trabalho, separando-se a concepção e execução, pela hierarquia, pela racionalização das estruturas administrativas e ênfase em procedimentos e rotinas, além da alienação do trabalhador quanto ao seu objeto de trabalho, o paciente. A comunicação é verticalizada, assim como o poder e a tomada de decisão, sendo que a equipe acata as ordens vindas da direção/gerência.

Nessa perspectiva, o enfermeiro, ao desempenhar as funções gerenciais, busca atender às metas institucionais e solucionar problemas de outros profissionais; assim, o serviço de enfermagem, geralmente é guiado pelas regras burocráticas, com maior valorização de normas e rotinas, fato que influencia a prática gerencial, nem sempre atendendo às necessidades de assistência ao paciente. 
Contudo, ressalta-se que para a viabilização dos princípios do Sistema Único de Saúde (SUS), tornam-se necessárias que as ações das instituições focalizem nas necessidades de saúde dos usuários. Nesse sentido, a integralidade do cuidado se apresenta como uma estratégia que possibilita conhecer a realidade e organizar a atenção à saúde. Para tanto, a identificação das prioridades, baseadas no perfil epidemiológico do território, permitiriam uma otimização dos recursos existentes entre os serviços. Trata-se da busca da universalidade de modo que se garanta o acesso não só para aqueles que procuram, mas também, para aqueles que mais precisam ${ }^{14}$.

Ainda, destaca-se que no âmbito hospitalar a integralidade da assistência no cuidado depende do trabalho e da colaboração da equipe, como também de uma boa articulação de suas práticas, porém a utilização deste princípio nessas instituições é de difícil realização, pois está associada a uma grande demanda espontânea do dia a dia do processo de trabalho ${ }^{14,15}$.

Com fins para alcançar a assistência integral, modelos de atenção assistenciais que respondam de forma eficiente todo o processo de organização do trabalho se fazem cruciais, ou seja, modelos que integrem a participação de várias categorias profissionais de forma a realizar um cuidado integral. A este respeito, pontuam-se modelos de atenção hospitalar como a clínica ampliada, onde o foco busca alcançar a integralidade do cuidado por meio do trabalho em equipe ${ }^{16}$.

Entretanto, é preciso destacar que para que um modelo de atenção efetivo se concretize, é imprescindível a associação de modelos de gestão participativos. Para a equipe de enfermagem, a implantação deste modelo irá possibilitar a aprendizagem coletiva, permitindo ainda a participação de todos na tomada de decisão.

Os modelos de gestão autoritários, com linhas de comando definidas, têm gerado insatisfação e conflito no trabalho da equipe de saúde ${ }^{17}$; assim faz-se necessária a urgente mudança de paradigma de forma a permitir a implementação de um modelo de gestão inovador que permita a participação de todos os profissionais ${ }^{18}$.

Modelos de gestão participativos, compartilhados preveem a reestruturação dos organogramas verticalizados e rígidos pautados na Abordagem clássica da administração. Um modelo de gestão descentralizado pressupõe a lateralização da comunicação e o achatamento do organograma. Assim, é previsto que a decisão seja diluída entre os diferentes integrantes da equipe interdisciplinar, a fim de apresentar informações mais completas, diversidade de experiências e perspectivas. Outras vantagens incluem o aumento da legitimidade, visto que a decisão conjunta e democrática faz com que o grupo se sinta comprometido e corresponsável pelas ações implementadas ${ }^{19}$.

Nessa perspectiva, trabalhar um modelo participativo exige a organização tanto do líder como de seus liderados. O enfermeiro, enquanto líder e gerente de sua equipe deve estar capacitado para atuar de forma acertada junto aos demais profissionais, contribuindo para o desenvolvimento de competências, auxiliando-os nas diversas ações que permeiam o cuidado do paciente no cenário hospitalar.

Neste contexto, a comunicação pode ser considerada ferramenta gerencial que depende da democracia. Diálogo e deliberação, como trocas autênticas entre as pessoas, não são baseadas no desempenho competitivo, mas sim em conversas desafiadoras que, muitas vezes, levam a um novo aprendizado pessoal e social ${ }^{20}$.

Outro aspecto relevante do modelo participativo de gestão é a descentralização de poder que garante a colaboração interprofissional. Ao concentrar-se na redução das disparidades e fornecendo ao pessoal oportunidades para expressar suas perspectivas, os líderes podem reduzir os diferenciais de poder entre os vários grupos profissionais $^{21,22}$

Apesar do previsto nesse modelo gerencial inovador, várias fragilidades podem ser encontradas na prática hospitalar, especialmente para a equipe de enfermagem. A comunicação diretiva ainda é vislumbrada em algumas instituições que adotaram o modelo colegiado. Além disso, a comunicação lateral ou interunidades é incipiente, dificultando a decisão coletiva ${ }^{18,23}$.

Nesse sentido, comunicação dialógica, processo decisório e poder descentralizados são princípios fundamentais para garantir a implantação de um modelo de gestão democrático e compartilhado no ambiente hospitalar.

\section{CONCLUSÃO}

O modelo tradicional de atenção está arraigado à vida institucional, uma vez que, apesar das inúmeras críticas, é um modelo que funciona até os dias atuais. Contudo, considerar suas limitações é essencial, fazendo-se necessária a transposição para um modelo que agregue o coletivo de trabalhadores, de modo que se sintam engajados e motivados por se considerarem corresponsáveis e fundamentais ao processo de trabalho.

No caso dos profissionais de enfermagem, que representam expressivo contingente de pessoas no contexto hospitalar, a mudança para um modelo de atenção que atenda o indivíduo na sua totalidade, gera a necessidade de um modelo de gestão mais participativo, com o envolvimento da equipe multiprofissional na tomada de decisão e resolução dos problemas, bem como na elaboração de propostas de melhorias, de forma que todos se sintam corresponsáveis pelas decisões. 
Mas, vale destacar que mudanças nos modelos de gestão tradicionais para modelos mais flexíveis, democráticos são difíceis de ocorrer, especialmente pela resistência daqueles que não compreendem o processo. Portanto, a persistência é essencial, assim como a continuidade das propostas, a utilização de estratégias educativas que visem o alcance efetivo de todos os pressupostos da gestão descentralizada; e o aprofundamento do conhecimento acerca desta temática para que haja a sustentação maior da prática por meio da teoria e do acúmulo de experiências dos trabalhadores envolvidos.

\section{REFERÊNCIAS}

1. Holanda FG, Marra CC, Cunha ICKO. Construction of a professional competency matrix of the nurse in emergency services. Acta Paul. Enferm. 2014 [cited 2019 Jun 12]; 27(4):3373-79. DOI: http://dx.doi.org/10.1590/1982-0194201400062

2. Cardoso IVCP. O mundo do trabalho após a doutrina neoliberal no Brasil: privatização e flexibilização no contexto de crise econômica. Rev. Pegada. 2016 [cited 2019 Jun 12]; 17(1):4-27. DOI: https://doi.org/10.33026/peg.v17i1.4403

3. Brasil. Ministério da Saúde (Br). Portaria no 3.390, de 30 de dezembro de 2013. Institui a Política Nacional de Atenção Hospitalar (PNHOSP) no âmbito do Sistema Único de Saúde (SUS). Brasília, (DF): Ministério da Saúde; 2013. [cited 2019 Jun 12]. Available from: http://www.saude.sp.gov.br/resources/humanizacao/biblioteca/leis/outras-relevantes/portaria__politica_nacional_de_atencao_hospitalar___ministerio_da_saude.pdf

4. Paim JA. Modelos de atenção e vigilância da saúde. In: Rouquayrol MZ, Almeida FN, organizadores. Epidemiol e Saúde. 6‥ ed. Rio de Janeiro (RJ): MEDSI; 2003. p. 567-86.

5. Santos TB, Pinto ICM. National policy for hospital care: con(di)vergences among the federal executive's rules, conferences and strategies. Saúde. debate. 2017 [cited 2019 Jun 12]; 41(3):99-113. DOI: http://dx.doi.org/10.1590/0103-11042017s308

6. Trindade LL, Pires DEP. Implications of primary health care models in workloads of health professionals. Texto \& contexto Enferm. 2013 [cited 2019 Jun 12]; 22(1):36-42. DOI: http://dx.doi.org/10.1590/S0104-07072013000100005

7. Leal LM, Castro MMC. Política nacional de atenção hospitalar: impactos para o trabalho do assistente social. Serv. Soc Saúde. 2017 [cited 2019 Jun 12]; 16(2):211-28. DOI: https://doi.org/10.20396/sss.v16i2.8651464

8. Foucault M. O nascimento do hospital. In: Foucault M. Microfísica do poder. 4a. ed. Rio de Janeiro: Graal; 2014. p. 99-112.

9. Goulart BF, Coelho MF, Chaves LDP. Nursing staff in hospital attention: integrative review. Rev. Enferm. UFPE on line. 2014 [cited 2019 Jun 12]; 8(2):386-395. Available from: https://periodicos.ufpe.br/revistas/revistaenfermagem/article/view/9686

10. Myrick D. Frederick Taylor as a contributor to public administration. Med. J. Social Scienc. 2012 [cited 2019 Jun 12]; 3(12):10-20. Available from: http://uir.unisa.ac.za/bitstream/handle/10500/8584/Myrick_Taylor\%20Article.pdf

11. Cassiano AN, Santos TR, Souza MB, Valença CN, Holanda CSM, Germano RM. The management of health services in the perspective of the humanist administration theory. Rev. enferm. UFPE on line. 2011 [cited 2019 Jun 12]; 5(8):2060-65. DOI: https://doi.org/10.5205/reuol.1262-12560-1-LE.0508201133

12. Weber M. Conceitos básicos de sociologia. Tradução de Rubens Eduardo Ferreira Frias e Gerard Georges Delaunay. São Paulo: Moraes; 1987.

13. Chaves LDP, Balderrama P, Margatho A, Alves LR, Goulart BF, Camelo SHH. Hospital and nursing from the perspective of health care networks. CuidArte. Enferm. 2016 [cited 2019 Jun 12]; 10(2):218-25. Available from: http://www.webfipa.net/facfipa/ner/sumarios/cuidarte/2016v2/218-225.pdf

14. Soares EB, Guanilo MEE, Amestoy SC, Santos BP, Ceolin T. Perception of nursing professionals in hospital units in what concerns user embracement. Espaço ciência \& saúde. 2018 [cited 2019 Jun 06]; 6(2):1-11. Available from: http://www.revistaeletronica.unicruz.edu.br/index.php/enfermagem/article/view/6929/1761

15. Oliveira CB, Santos ATS, Andrade ASA, Gallotti FCM. A integralidade do cuidado à saúde sob a ótica do acadêmico de enfermagem. Int. Nurs Congress. 2017 [cited 2019 Jun 12]; 1(1):1-4. Available from: https://eventos.set.edu.br/index.php/cie/article/view/6020

16. Kalichman AO, Ayres JRCM. Comprehensiveness and healthcare technologies: a narrative on conceptual contributions to the construction of the comprehensiveness principle in the Brazilian Unified National Health System. Cad. Saúde Pública. 2016 [cited 2019 Jun 12]; 32(8):e00183415. http://dx.doi.org/10.1590/0102-311X00183415

17. Cramm JM, Strating MMH, Nieboer AP. The influence of organizational characteristics on employee solidarity in the long-term care sector. J. Adv Nurs. 2013 [cited 2019 Jun 12]; 69(3):526-34. DOI: http://dx.doi.org/10.1111/j.1365-2648.2012.06027.x

18. Bernardes A, Cummings G, Gabriel CS, Martinez-Évora YD, Coleman-Miller G. Implementation of a participatory management model: analysis from a political perspective. J. Nurs. Manag. 2015 [cited 2019 Jan 12]; 23(7):888-97. DOI: http://dx.doi.org/10.1111/jonm.12232

19. Hayashida KY, Bernardes A, Mazieiro VG, Gabriel CS. Decision-making of the nursing team after the revitalization of a decentralized management model. Texto \& contexto enferm. 2014 [cited 2019 Jun 12]; 23(2):286-93. DOI: http://dx.doi.org/10.1590/010407072014001190013

20. Raelin JA. Dialogue and deliberation as expressions of democratic leadership in participatory organizational change. J Org Change. 2012 [cited 2019 Jun 12]; 25(1):7-23. DOI: https://doi.org/10.1108/09534811211199574

21. Petri L. Concept analysis of interdisciplinary collaboration. Nurs. Forum. 2010 [cited 2019 Jun 12]; 45(2):73-82. DOI: http://doi.org/10.1111/j.1744-6198.2010.00167.x

22. Filho SAM, Souza NVDO, Gonçalves FGA, Pires AS, Varella TCMYML. Micro-powers in the daily work of hospital nursing: an approximation to the thinking of Foucault. Rev. enferm. UERJ. 2018 [cited 2019 Oct 23]; 26:e30716. DOI: http://dx.doi.org/10.12957/reuerj.2018.30716

23. Simões TR, Vannuchi MTO, Rossaneis MA, Silva LG, Haddad MCL, Jenal S. Continuing education as conceived by nurses in a highcomplexity philanthropic hospital. Rev. enferm. UERJ. 2013 [cited 2019 Jun 12]; 21(esp1):642-7. Available from: https://www.epublicacoes.uerj.br/index.php/enfermagemuerj/article/view/10042/8069 